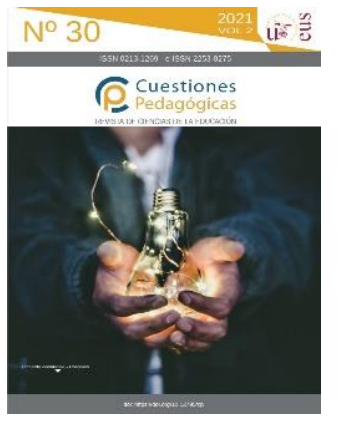

\title{
Factores determinantes del emprendimiento femenino en España: Identificación y análisis de una realidad
}

\author{
Key factors of female entrepreneurship in Spain: \\ identification and analysis of the situation
}

Recibido: 29/09/2021 | Revisado: 11/10/2021 | Aceptado: 26/11/2021 | Online first: 30/11/2021 Publicado: 31/12/2021

\author{
Ángela Martín-Gutiérrez \\ Universidad Internacional de La Rioja y Universidad de Sevilla \\ angela.martin@unir.net-amartin9@us.es \\ https://orcid.org/0000-0001-9847-245X
}

\section{Carolina Fernández-Salinero Miguel}

Universidad Complutense de Madrid

cfernand@ucm.es

https://orcid.org/0000-0003-3478-9049

\section{Beatriz De La Riva-Picatoste}

Universidad Complutense de Madrid

beariva@ucm.es

https://orcid.org/0000-0002-5719-9538

Resumen: El emprendimiento es uno de los motores de la economía a nivel internacional. Su visibilidad desde la perspectiva de género, sin embargo, no se produce hasta los años noventa del siglo pasado, siendo a partir de esa década cuando aparecen trabajos que analizan el emprendimiento femenino. Este artículo es heredero de ese interés y, desde la teoría fundamentada como metodología cualitativa de trabajo, se plantea identificar los factores determinantes del emprendimiento femenino y visibilizar la influencia de estos factores en España (con apoyo en los Informes GEM). Los resultados muestran tres bloques de factores determinantes del emprendimiento femenino: estructurales y sociales; cognitivos y emocionales; e impulsores/ bloqueadores. Con relación al primero, se descubre mayor presencia de empresas femeninas iniciadas en los sectores de servicios al consumidor y empresa, que requieren una inversión menor, favoreciendo su consolidación. En el segundo bloque, la
Abstract: Entrepreneurship is one of the driving forces of international economy. Its visibility from a gender-oriented point of view, however, does not occur until the nineties of the last century, when works analyzing female entrepreneurship first appeared. This article takes on this interest and, from the grounded theory as a qualitative methodology, aims to identify the key factors of female entrepreneurship as well as to make visible their influence in Spain (backed up by GEM Reports). Results show three kinds of determinant factors of female entrepreneurship: structural and social; cognitive and emotional; and drivers/blockers. Concerning the former, there is a greater presence of women's businesses that started in the sectors of consumer services and enterprise, which require less investment, thus promoting their consolidation. For the second type, "wealth creation" has been found to be the main motivation among young companies, which undertake mostly by means of opportunity, while "making a living owning because the lack of 
"creación de riqueza" es la primera motivación de las empresas jóvenes que emprenden más por oportunidad, y "ganarse la vida debido a la escasez del trabajo" es la aspiración principal de las maduras que emprende más por necesidad. En el último bloque, se evidencia que el marco institucional (financiación e internacionalización), social (redes), empresarial (tipo de empresa, innovación, uso y novedad tecnológica) y familiar (tradición y apoyo) son factores impulsores/bloqueadores del emprendimiento femenino. Se realiza finalmente una breve prospectiva de futuro del emprendimiento femenino. work" is the main aspiration of mature women who undertake primarily by necessity. Regarding the last set, it is evident that the institutional framework (financing and internationalization), social (networks), business (type of company, innovation, use and technological novelty) and family (tradition and support) are drivers/blockers of female entrepreneurship. Finally, a brief perspective on the future of female entrepreneurship is set out.
Palabras clave: emprendimiento, género, factores de emprendimiento, tipos de emprendimiento, fases del emprendimiento, informes GEM.
Keywords: Entrepreneurship, gender, entrepreneurship factors, types of entrepreneurship, stages of entrepreneurship, GEM report.

\section{Introducción ${ }^{1}$}

El emprendimiento es un importante motor de la economía a nivel internacional. Resulta fundamental para el crecimiento económico, la renovación del tejido empresarial, la creación de puestos de trabajo, la difusión de los avances científicos y el desarrollo territorial (Tejeiro-Koller et al., 2021). La literatura económica estudia los procesos de emprendimiento desde diferentes perspectivas, y una de ellas es la de género. Sin embargo, hasta los años noventa del siglo pasado la invisibilidad de la mujer emprendedora era notoria en la literatura económica, siendo a partir de esa década cuando esta tendencia empieza a cambiar y aparecen los primeros trabajos que analizan el emprendimiento femenino (Montero-González y CamachoBallesta, 2018).

Para poder abordar este tema, de entre las posibles clasificaciones existentes nos apoyamos en aquella que identifica tres tipos de factores determinantes para entender el emprendimiento en clave de género: estructurales y sociales, cognitivoemocionales e impulsores y bloqueadores. Su identificación y análisis resulta esencial para conseguir una visión global del emprendimiento femenino actualmente y es la base para visibilizar la situación de las emprendedoras en España, así como para

\footnotetext{
${ }^{1}$ Este artículo está vinculado al proyecto «Formación del Potencial Emprendedor. Generación de un Modelo Educativo de Identidad Emprendedora», con Referencia PID2019-104408GB-I00, dentro del Plan Estatal 2017-2020 de Proyectos I+D+i (Generación de Conocimiento), financiado por: Ministerio de Ciencia e Innovación - Agencia Estatal de Investigación/10.13039/501100011033.
} 
estudiar los tipos de emprendimiento femenino (por necesidad y por oportunidad) y sus fases de desarrollo (potencial, joven y consolidada).

Finalizamos este trabajo con un planteamiento prospectivo del emprendimiento femenino mediatizado por la pandemia debida al COVID 19, que va a requerir una reactivación y transformación del emprendimiento, especialmente del femenino, promoviendo iniciativas económicas, sociales, educativas y políticas.

\section{Objetivos}

Tres son los objetivos que planteamos en este trabajo:

1) Identificar los factores determinantes del emprendimiento femenino.

2) Visibilizar la influencia de los factores determinantes del emprendimiento femenino en España a través del análisis de los Informes GEM más actuales a los que hemos tenido acceso.

3) Realizar una prospectiva de futuro del emprendimiento femenino.

\section{Metodología e instrumentos}

La metodología que nos sirve de base para conseguir dichos objetivos se apoya en lo que Bisquerra (2009) denomina "teoría fundamentada", un método cualitativo de investigación de naturaleza exploratoria, cuyo propósito es descubrir conceptos y proposiciones partiendo directamente de los datos, realizando un análisis descriptivo-interpretativo que utiliza la técnica del análisis documental.

Los instrumentos que empleamos bajo este paraguas metodológico son los informes publicados por GEM (Global Entrepreneruship Monitor) España entre los años 2017 y 2020. Los cuales nos aportan datos concretos y actualizados de aspectos que consideramos de especial relevancia en este trabajo como son los factores determinantes del emprendimiento femenino, los tipos de emprendimiento femeninos existentes y sus fases de desarrollo.

\section{El emprendimiento femenino}

Para estudiar este tema debemos identificar aquellos factores que determinan el emprendimiento según el género de quien toma la iniciativa y su singularidad (García-Villalobos et al., 2019). Barney (1991) plantea cuatro tipos de factores capaces de promover emprendimiento (valiosos, raros, difícilmente imitables y sustituibles). Galindo y Méndez (2011), por su parte, señalan como factores importantes el entorno social, la familia, la cultura de origen y las expectativas sobre la actividad económica del país. Otros factores que impulsarían el éxito del emprendimiento, según De la O Cordero y Monge-González (2019), serían la disciplina, la educación, el acompañamiento y la financiación. Pero la clasificación 
que nos parece más adecuada es la propuesta por Montero-González y CamachoBallesta (2018), cuya adaptación se muestra a continuación (ver Tabla 1).

Tabla 1

Factores determinantes del emprendimiento femenino

\begin{tabular}{ccc}
\hline $\begin{array}{c}\text { Factores estructurales y } \\
\text { sociales }\end{array}$ & $\begin{array}{c}\text { Factores cognitivos y } \\
\text { emocionales }\end{array}$ & $\begin{array}{c}\text { Factores impulsores } \mathbf{y} \\
\text { bloqueadores }\end{array}$ \\
\hline Objetivos de negocio & Autoeficacia & Entorno contextual-familiar \\
Sector & Estereotipos & Redes sociales \\
Formación & Percepción & Acceso a la financiación \\
Experiencia previa & Miedo al fracaso & Innovación \\
& Motivaciones y aspiraciones & Marco institucional \\
\hline
\end{tabular}

Fuente. Adaptación de Montero-González y Camacho-Ballesta (2018, p.42).

Al analizar el contenido de la Tabla 1 identificamos tres tipos de factores determinantes del emprendimiento femenino. Los estructurales y sociales, que nos aportan información acerca de los objetivos de negocio, los sectores de actividad, la formación en emprendimiento y la experiencia profesional previa. Los cognitivos y emocionales que inciden en aquellos aspectos personales que incitan a las mujeres a emprender. $\mathrm{Y}$, los impulsores y bloqueadores, que son las ventajas e inconvenientes a los que debe enfrentarse el emprendimiento femenino. A continuación, vamos a pararnos en cada uno de ellos más detenidamente.

\section{Factores estructurales y sociales del emprendimiento femenino}

Diversos autores han analizado las diferencias de género que afectan al comportamiento empresarial (Brush, 1992; Brüderl et al., 1992; Themudo, 2009). Brush (1992) observó que una de las más significativas se centra en los objetivos de negocio. Mientras que los hombres se identifican fundamentalmente con los objetivos económicos, las mujeres se orientan prioritariamente al bien común. Esto hace que algunas investigaciones sitúen a las mujeres como objetivo clave del emprendimiento social (Themudo, 2009; García-Villalobos et al., 2019).

Por otra parte, la elección del sector para el desarrollo de la actividad emprendedora es también un factor que genera bastantes diferencias entre hombres y mujeres. Esta elección condiciona las características del emprendimiento femenino, pero afecta especialmente a la dimensión de sus negocios, a su crecimiento e internacionalización y al acceso a fuentes de financiación, lo que supone un importante freno para desarrollar actividades en sectores que requieren fuertes inversiones (tecnológicos, determinados tipos de comercio, agrícolas,...), precisamente aquellos en los que las mujeres tienen menor presencia. Asimismo, la ausencia de equilibrio en la conciliación de la vida familiar y profesional es uno de los elementos que puede forzar al emprendimiento femenino a orientarse hacia sectores que requieren una dedicación de menor intensidad (Montero-González y CamachoBallesta, 2018). 
Respecto a la formación, existen investigaciones que muestran que las emprendedoras con un mayor nivel educativo obtienen un mejor desempeño, ya que la diferencia en los resultados disminuye sustancialmente cuando las mujeres poseen estudios universitarios (Elizundia, 2014). Sin embargo, según la OCDE (2013) no es tanto el nivel educativo, sino más bien el tipo de educación lo que realmente importa en el emprendimiento femenino, puesto que las mujeres están subrepresentadas en aquellos estudios que desarrollan conocimientos útiles para la creación de empresas (economía, comercio, informática, tecnología), lo que repercute en la creación de negocios pequeños, en la elección de sectores de actividad no demasiado innovadores o en el desarrollo de un emprendimiento más de necesidad, concebido como actividad básica de autoempleo, que de oportunidad, asociado a la creación de negocios innovadores y startups.

En relación con la experiencia previa, debemos diferenciar entre experiencia profesional previa, en puestos directivos y en el sector. En lo relativo a la primera, la falta de experiencia profesional previa puede ser un freno para reconocer oportunidades de negocio y para el desarrollo de la actividad emprendedora. Brüderl et al. (1992) afirman que las empresarias suelen tener menos años de experiencia laboral previa, lo que puede disminuir sus posibilidades de éxito futuro. Con relación a la experiencia en puestos directivos, se observa la existencia de segregación vertical en el mercado laboral, lo que provoca que un porcentaje reducido de mujeres tenga acceso a puestos de alta dirección (el denominado "techo de cristal"), invisibilizando su potencial y desaprovechando su talento (Montero-González y Camacho-Ballesta, 2018). Un mayor equilibrio de género en la alta dirección puede, a este respecto, tener importantes efectos de "derrame" (spillover) sobre el emprendimiento femenino. Finalmente, el número de mujeres que conocen el sector antes de iniciar su actividad emprendedora es menor al de hombres, y aquellas que cuentan con esa experiencia, inician más tarde sus negocios (OCDE, 2013).

\section{Factores cognitivos y emocionales del emprendimiento femenino}

La acción de emprender depende en gran medida de la autoeficacia de las mujeres, es decir, de la conciencia que tienen de poseer la competencia necesaria para poner en marcha una actividad. Y también de los estereotipos de género, los cuales influyen en el diferente desarrollo de la percepción que tienen hombres y mujeres respecto de sus competencias y preferencias por crear un determinado negocio (Zhao et al., 2005), aunque entre las mujeres emprendedoras se van superando, paulatinamente, estos estereotipos sociales, presentando capacidades y percepciones cada vez más similares a las de los hombres (DeTienne y Chandler, 2007). Asimismo, la decisión de emprender suele generar incertidumbre. Si la persona emprendedora tiene miedo y percibe que la opción de crear una empresa es demasiado arriesgada, no seguirá adelante. Ese miedo al fracaso como obstáculo para emprender suele tener mayor incidencia en el género femenino (Arenius y 
Minniti, 2005). Y en cuanto a las motivaciones y aspiraciones de las emprendedoras, destacan como las más comunes, la obtención de independencia, la necesidad de logro, la ausencia de trabajo por cuenta ajena o la falta de recursos para la subsistencia, entre otras, las cuales mediatizan que el emprendimiento sea por necesidad o por oportunidad (Santander-Astorga et al., 2016).

\section{Factores impulsores y bloqueadores del emprendimiento femenino}

El último grupo de factores a los que nos vamos a referir son aquellos que facilitan o dificultan el emprendimiento femenino (Álvarez et al., 2013; Álvarez et al., 2012). Comenzaremos con el entorno contextual-familiar, que hace referencia a la comunidad que rodea a la mujer emprendedora (estilo de vida, cultura, tipo de negocios, expectativas de crecimiento económico), al área o zona en la que se ubica (demografía, organización social), así como a los apoyos morales y económicos que ofrece la familia o la tradición emprendedora de la misma. Las condiciones del contexto nacional y las del contexto político, social y educativo se conjugan así para crear el ambiente que promueve una actividad emprendedora orientada al crecimiento económico (Maldonado et al., 2016).

Por otro lado, desde los años 90 del siglo pasado el número de estudios orientados a analizar la relación entre las redes sociales y la actividad emprendedora se ha incrementado notablemente (Hoang y Antoncic, 2003; Gordon y Jack, 2010). La investigación ha encontrado evidencias de la estrecha relación existente entre el éxito de una persona emprendedora y su capacidad para desarrollar redes sociales que incrementen el número de recursos valiosos para reconocer oportunidades en el mercado, el acceso a fuentes de información relevantes para el negocio o la posibilidad de supervivencia de la empresa. Además, las redes sociales pueden aportar la legitimidad y el apoyo emocional necesarios para sobrevivir durante las primeras etapas del negocio (Montero-González y Camacho-Ballesta, 2018). En este sentido, las mujeres se encuentran en desventaja frente a los hombres al carecer de redes sociales adecuadas y eficientes. Esto se debe a que las redes sociales de las mujeres son más reducidas, menos diversas y en ellas predominan las relaciones familiares (Díaz y Carter, 2009). Por ello, se hace necesario promover redes específicas sobre emprendimiento como la "Red de Embajadores" de la Comisión Europea, constituida por personas emprendedoras exitosas que inspiran a mujeres de todas las edades a establecer sus propios negocios (OCDE, 2013).

El acceso a financiación es otro factor a tener en cuenta. Diferentes investigaciones han puesto de relieve que las mujeres tienden a acceder a menos fuentes de financiación para sus negocios que los hombres, entre otras razones, por miedo al riesgo, por no perder el control de su negocio o por las prácticas abusivas de las entidades financieras (Heilbrunn, 2004; García-Villalobos et al., 2019). De hecho, la financiación es el obstáculo principal en el emprendimiento femenino el cual, aunque no pueda probarse como un factor de discriminación, parece estar 
relacionado con el tamaño de las empresas que crean las mujeres (por lo general pequeñas), que ofrecen menos garantías de éxito a las entidades financieras (Álvarez et al., 2012).

El último factor considerado en este análisis es el marco institucional de apoyo a la actividad emprendedora, que englobaría a las políticas de emprendimiento nacionales y regionales encaminadas a apoyar a las emprendedoras. El estudio de este factor pone de manifiesto el desconocimiento de las mujeres de la existencia de este tipo de instrumentos de apoyo, lo que hace necesario que se creen más políticas para aumentar la conciencia del emprendimiento como una opción profesional para las mujeres. Pero no solo deben ponerse en marcha políticas de iniciativa empresarial que se orienten a la creación de empresas, sino que deben ampliarse con instrumentos que puedan a ayudar a las empresas femeninas ya consolidadas a alcanzar sus aspiraciones de crecimiento (OCDE, 2013).

\section{Los factores determinantes del emprendimiento femenino en España a través del análisis de los Informes GEM}

El análisis realizado de los factores determinantes del emprendimiento femenino nos proporciona un marco teórico general que vamos a cotejar con la realidad del emprendimiento femenino en España. Para ello, nos apoyamos en el Global Entrepreneurship Monitor (GEM), una destacada red mundial sobre emprendimiento que tiene como objetivo divulgar información sobre la iniciativa emprendedora. Con la finalidad de ofrecer cifras contrastadas, en este trabajo vamos a realizar un análisis de los informes publicados por GEM España entre los años 2017 y 2020, a fin de extraer de los mismos información relevante que se adecúe a la base teórica que aquí se plantea. Completando dichas fuentes con los informes monográficos anuales proporcionados también por GEM.

El objetivo final es visibilizar cómo se comporta el emprendimiento femenino en España, tanto por necesidad como por oportunidad. Comprobando hasta qué punto los factores determinantes señalados inciden en el emprendimiento de las mujeres, tanto en su fase inicial de creación de negocio, como en la de consolidación del mismo.

\section{Tipología del emprendimiento femenino: la situación en España}

Desde el año 2005, la Tasa de Emprendimiento Masculino (TEA masculina) ha oscilado entre un mínimo del 5,4\% (2010) y un máximo del 9,7\% (2007), mientras que la TEA femenina ha variado entre un mínimo del 3,9\% (2009) y un máximo del $6 \%$ (2018) (Peña et al., 2019). Estos datos van arrojando cierto optimismo a medida que avanzan los años, pues se observa que la brecha de género va disminuyendo y, ya en el año 2018 , el $53,1 \%$ de las personas emprendedoras eran hombres frente al 
46,9\% que eran mujeres. Sin embargo, según el Mapa del Emprendimiento en España, que muestra las consolidaciones del ecosistema emprendedor nacional (South Summit, 2019), las mujeres representan solo el 19\% de la población emprendedora, aunque el $39 \%$ de las startups españolas tengan al menos una mujer en el equipo fundador. Además, el $62 \%$ de las mujeres emprende sola. El informe GEM España 2018-2019 (Peña et al., 2019) señala también que las mujeres tienen mayores probabilidades de éxito en sus negocios, lo que puede deberse a que buscan fuentes de financiación más sólidas, a pesar de encontrarse con más dificultades para acceder a las mismas (Figueroa et al., 2011).

Estos datos muestran al emprendimiento femenino en general, pero ¿hay diferencias en función de su tipología? A continuación, vamos a analizar cómo influye esta situación según el tipo de emprendimiento.

\section{Emprendimiento por necesidad}

Si nos apoyamos en el Informe GEM España 2018-2019 (Peña et al., 2019), último informe GEM en el que aparece la distinción entre emprendimiento por necesidad y emprendimiento por oportunidad, descubrimos que una persona emprendedora por necesidad es aquella que se encuentra inmersa en el proceso emprendedor por no tener una mejor opción en el mercado laboral, viéndose determinada por las circunstancias o por la falta de expectativas. Así, el emprendimiento por necesidad se debe a estimulaciones relacionadas con la motivación de logro, el deseo de independencia y la búsqueda de desarrollo social (Verheul et al., 2016). Este tipo de emprendimiento hace que la persona se sienta forzada a montar una empresa, pero sin cubrir un hueco del mercado (Aido-Almagro et al., 2016). Aparece entonces un tipo de emprendimiento de bajo potencial, con mayor tendencia al fracaso que el emprendimiento por oportunidad (Terán y Guerrero, 2019) y con más posibilidades de mortalidad (Peña et al., 2019). Es necesario destacar a este respecto que las tasas de actividad emprendedora se han visto incrementadas en el emprendimiento impulsado por necesidad desde 2015, con unos porcentajes de entre el $22,6 \%$ y el $28,3 \%$, mientras que en el año 2009 rondaba el 15\% (Peña et al., 2019).

Los factores cognitivos y emocionales son los que resultan más determinantes en este tipo de emprendimiento. De ellos, la motivación y las aspiraciones son los fundamentales; destacando como el principal motivo de las mujeres para emprender el de "ganarse la vida", aunque con un porcentaje de interés ligeramente superior al de los hombres $(74,10 \%$ frente a $70,70 \%)$, según se recoge en el Informe GEM España 2019-2020. Respecto al deseo de gozar de una situación de mayor riqueza, existe un interés mucho menor en mujeres que en hombres $(27,60 \%$ frente a $41 \%)$ (Peña et al., 2020). 
Sin embargo, las brechas de género han ido disminuyendo, pues al retomar los datos del Informe GEM España 2017-2018 (Peña et al., 2018), podemos comprobar que en el año 2017 el porcentaje de población general entre 18 y 64 años que realizó algún tipo de emprendimiento por necesidad se situó en el $0,9 \%$, suponiendo el $28,3 \%$ sobre el número total de las personas emprendedoras; identificando que de cada 10 personas que iniciaron algún tipo de emprendimiento, $5 / 6$ eran hombres frente a $4 / 6$ que eran mujeres. Según indica el mismo informe, un año después, la actividad emprendedora total creció un 3,2\%, disminuyendo el 20,3\% el emprendimiento por necesidad, lo que puede ser debido a que el emprendimiento por oportunidad comienza a ganar terreno.

\section{Emprendimiento por oportunidad}

Una persona emprendedora por oportunidad es aquella que escoge crear una empresa basándose en la percepción de que existe una oportunidad de negocio que cree rentable y que no está siendo aprovechada o lo está siendo de forma incompleta por las empresas existentes (Peña et al., 2019). En este caso, los factores determinantes son fundamentalmente estructurales y sociales.

Las iniciativas emprendedoras por oportunidad presentan mayores índices de supervivencia que las debidas a necesidad (Peña et al., 2019), ya que las emprendedoras buscan oportunidades reales de negocio, aumentando así sus posibilidades de permanencia en el mercado (Aido-Almagro et al., 2016). Los principales objetivos de las mujeres para emprender son la posibilidad de mayor independencia (50,8\% de la actividad emprendedora total) y de aumentar los ingresos (32,4\% de la actividad emprendedora total) (Peña et al., 2020). Según el Informe GEM España 2017-2018 (Peña et al., 2018), en el año 2017 el porcentaje de población entre 18 y 64 años que realizó algún tipo de emprendimiento por oportunidad se situó en el $1,8 \%$, representando el $68,5 \%$ del número total de personas emprendedoras. Tan solo un año después, la cifra de personas emprendedoras motivadas por oportunidad se alzó hasta el $4,5 \%$ (de ellas, el $0,87 \%$ son mujeres), lo que representa el 70,7\% de la actividad total emprendedora (Peña et al., 2019).

\section{Fases de desarrollo de las empresas españolas desde la perspectiva de género}

Al analizar el emprendimiento desde la perspectiva de género, observamos que las diferencias entre mujeres y hombres existen también en función de la fase de desarrollo en la que se encuentre la empresa. Para entender mejor esta situación necesitamos abordar las tres fases del proceso emprendedor (Peña et al., 2020):

- Empresas potenciales, que son aquellas en las que una idea de negocio es viable económicamente. 
- Empresas jóvenes, que son las empresas nacientes, nuevas e iniciadas, las cuales están comenzando y, por tanto, sus beneficios no superan la inversión económica realizada.

- Empresas maduras o consolidadas, que son aquellas con experiencia en el mercado y económicamente solventes para poder pagar salarios.

Figura 1

Porcentaje de hombres y mujeres en las fases del proceso emprendedor

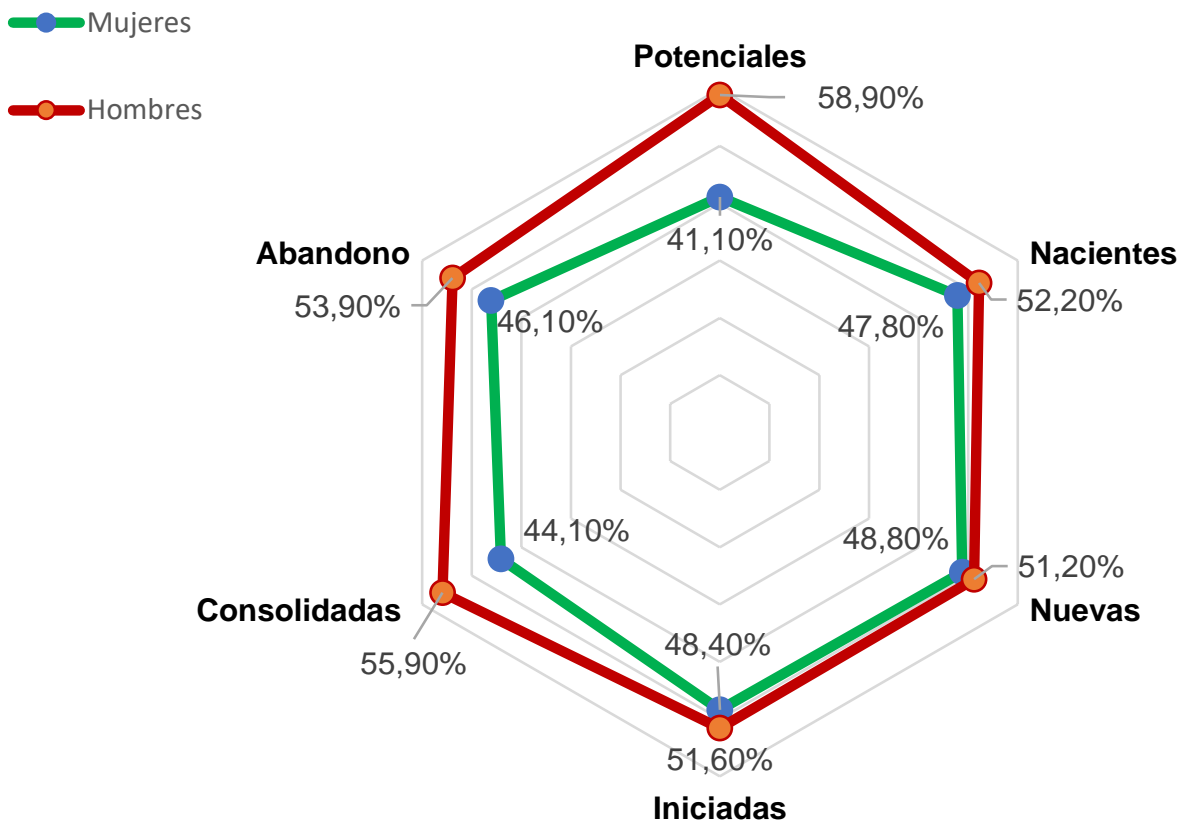

Fuente. Elaboración propia a partir del informe GEM España 2019-2020

En la Figura 1 se muestra el porcentaje de emprendedoras y emprendedores en el entorno español en función del proceso emprendedor en el que se encuentran. Cabe destacar que existe una mayor proporción de hombres que de mujeres en todo el proceso emprendedor: en las empresas potenciales (58,9\% frente a $41,10 \%)$, en las empresas jóvenes (nacientes, nuevas e iniciadas) y en las empresas consolidadas (55,9\% frente a 44,1\%). Esta situación evidencia que las mujeres tienen una menor presencia en el trabajo por cuenta propia y menos continuidad en el proceso emprendedor, ya que abandonan sus empresas en un 46,1\% (Peña et al., 2020).

\section{Factores determinantes del emprendimiento femenino según las fases del proceso emprendedor}

Vamos a analizar ahora el correlato que existe entre los factores cognitivoemocionales, estructurales y sociales del emprendimiento femenino, concebidos como impulsores o bloqueadores, y las fases del proceso emprendedor. 


\section{Factores cognitivo-emocionales del emprendimiento femenino y proceso emprendedor}

Como se muestra en la Figura 2, las motivaciones y aspiraciones de las emprendedoras son diferentes según nos refiramos a empresas jóvenes o consolidadas. En el primer caso, las emprendedoras destacan que sus motivaciones para emprender se basan en la creación de riqueza o en poseer una renta alta (nacientes $55,6 \%$ - nuevas $61,9 \%$ - iniciadas $59,5 \%$ ) y en marcar la diferencia en el mundo (nacientes $51,3 \%$ - nuevas $48,3 \%$ - iniciadas $49,4 \%$ ). Sin embargo, cuando la empresa es más madura o consolidada, se observa que hay más emprendedoras que han accedido a su creación para ganarse la vida $(71,20 \%)$ que para crear riqueza 0 poseer una renta alta (32\%). Podemos afirmar a este respecto que las mujeres de empresas jóvenes emprenden más por oportunidad que por necesidad y que las mujeres de empresas maduras mantienen su negocio más por necesidad que por oportunidad.

\section{Figura 2}

Aspiraciones y motivaciones en el emprendimiento femenino

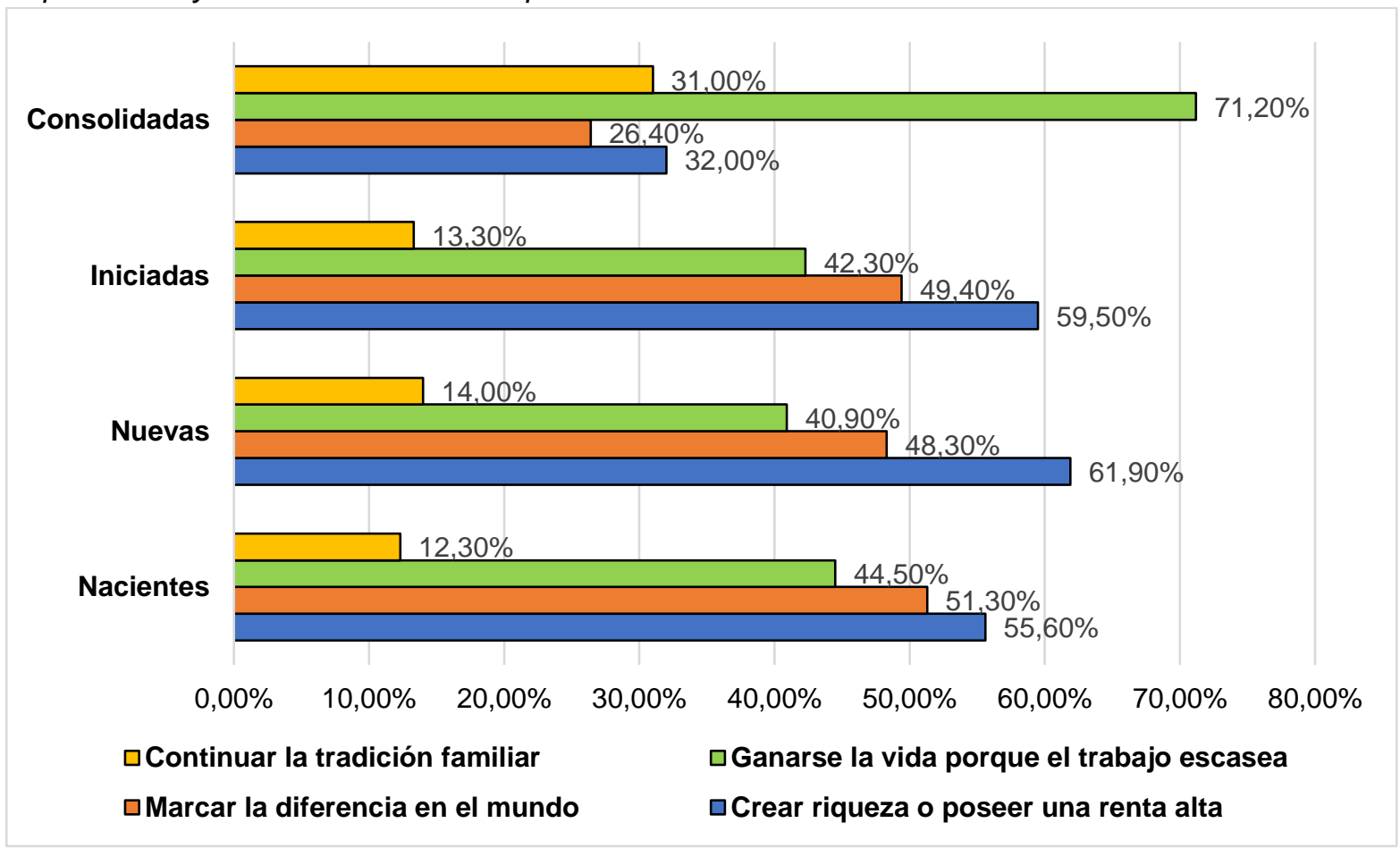

Fuente. Elaboración propia a partir del informe GEM España 2019-2020

La Figura 2 muestra también que las empresarias con un emprendimiento reciente dejan como última opción, dentro de sus motivaciones, continuar con la tradición familiar (nacientes 12,30\% - nuevas 14\% - iniciadas 13,30\%) y, sin embargo, es de las opciones más valoradas para las emprendedoras consolidadas (31\%). Una razón que puede apoyar estos datos es que gran parte de las empresas consolidadas sean negocios familiares heredados. Aguilar et al. (2020) afirman que cuando se hereda una empresa se es menos propensa a iniciar un nuevo negocio o a trabajar 
por cuenta ajena, ya que por tradición familiar la persona ya está trabajando en dicha empresa y, por tanto, continua con su consolidación.

\section{Factores socio-estructurales del emprendimiento femenino y proceso emprendedor}

Además de los factores cognitivos-emocionales señalados, la actividad emprendedora femenina tiene una influencia directa del contexto de la persona que desea emprender o que se encuentra emprendiendo. La generación del capital social, humano e incluso financiero, unido al acceso a diferentes recursos se produce gracias a los factores estructurales y sociales que poseen las mujeres. En este sentido, el marco institucional, empresarial y familiar se convierte en factor impulsor del emprendimiento femenino e incide en el tipo y en el sector de las empresas iniciadas y consolidadas, como se puede comprobar en la Tabla 2.

Tabla 2

Sectores de actividad emprendedora desde la perspectiva de género

\begin{tabular}{llrrrr}
\hline \multirow{2}{*}{$\begin{array}{l}\text { Tipos de } \\
\text { empresa }\end{array}$} & Género & Extractivo & Transformador & $\begin{array}{l}\text { Servicios a } \\
\text { empresa }\end{array}$ & $\begin{array}{l}\text { Servicios al } \\
\text { consumidor }\end{array}$ \\
\cline { 3 - 6 } Iniciadas & Mujer & $5,10 \%$ & $14,80 \%$ & $27,10 \%$ & $\mathbf{5 2 , 9 0 \%}$ \\
\cline { 2 - 6 } & Hombre & $3,80 \%$ & $24,10 \%$ & $\mathbf{4 0 , 3 0 \%}$ & $31,80 \%$ \\
\hline Consolidadas & Mujer & $10,20 \%$ & $12,90 \%$ & $19,90 \%$ & $\mathbf{5 7 \%}$ \\
\cline { 2 - 5 } & Hombre & $9,10 \%$ & $30,70 \%$ & $25 \%$ & $\mathbf{3 5 , 3 0 \%}$ \\
\hline
\end{tabular}

Fuente. Elaboración propia a partir del informe GEM España 2019-2020

Los datos de la Tabla 2 nos indican que las empresas jóvenes dirigidas por mujeres se encuentran principalmente en el sector de servicios al consumidor $(52,90 \%)$, seguidas del sector de servicios a empresa $(27,10 \%)$. Esto significa que el $80 \%$ de las empresas femeninas iniciadas se sitúan en sectores que requieren de una inversión económica más asumible y viable y que, gracias a ello, se mantienen en el tiempo hasta llegar a consolidarse (Arcos, 2020), puesto que esos mismos sectores son los más representados en las empresas consolidadas (57\% sector de servicios al consumidor y $19,9 \%$ servicios a empresa).

Requiere una mención especial el sector extractivo, aquel que está vinculado a la producción de materias primas para la construcción y para otras industrias, ya que a pesar del bajo porcentaje de emprendimiento que existe en el mismo, independientemente del tipo de empresa, cuenta con más mujeres que hombres, tanto en las empresas consolidadas $(10,20 \%$ frente al $9,10 \%)$ como en las iniciadas $(5,10 \%$ frente al 3,80\%). En estas últimas casi duplican el porcentaje las mujeres. Esta situación podría deberse al impulso, desde el ámbito educativo y formativo, de las competencias STEM (en español CTIM - Competencias vinculadas a las Ciencias, Tecnología, Ingeniería y Matemáticas). Macías et al. (2021) afirman que cada vez más el mercado laboral está demandando personas con estas 
competencias, proponiendo que se impulsen estrategias políticas, sociales y educativas que las desarrollen.

\section{Innovación, tecnología e internacionalización de las empresas}

Dedicamos un apartado específico al grado de innovación, uso y novedad de las tecnologías e internacionalización de las empresas españolas, tanto iniciadas como consolidadas, que son factores impulsores o bloqueadores del emprendimiento femenino.

\section{El emprendimiento desde la innovación}

El emprendimiento que se apoya en la innovación es un emprendimiento de oportunidad que se caracteriza por introducir ideas nuevas o mejoras de productos y servicios mediante la incorporación de diferentes tecnologías, prácticas de trabajo, formas de hacer negocios o procesos productivos (Vélez-Romero y Ortiz-Restrepo, 2016).

Como observamos en la Tabla 3, la innovación no es un indicador que caracterice a las empresas españolas, ya que más de un $60 \%$ de las nuevas empresas y más de un $80 \%$ de las empresas consolidadas no son innovadoras.

Tabla 3

Grado de innovación de las empresas españolas desde la perspectiva de género

\begin{tabular}{llccc}
\hline \multirow{2}{*}{$\begin{array}{c}\text { Tipo de } \\
\text { empresa }\end{array}$} & Género & \multicolumn{3}{c}{ Grado de innovación } \\
\cline { 3 - 5 } & & $\begin{array}{c}\text { Completamente } \\
\text { innovadora }\end{array}$ & $\begin{array}{c}\text { Algo } \\
\text { innovadora }\end{array}$ & No innovadora \\
\hline \multirow{2}{*}{ Iniciadas } & Mujer & $15 \%$ & $19,1 \%$ & $65,9 \%$ \\
\cline { 2 - 5 } & Hombre & $10,2 \%$ & $25,8 \%$ & $63,9 \%$ \\
\hline \multirow{2}{*}{ Consolidadas } & Mujer & $6,4 \%$ & $9,8 \%$ & $83,8 \%$ \\
\cline { 2 - 5 } & Hombre & $6,2 \%$ & $10,7 \%$ & $83,1 \%$ \\
\hline
\end{tabular}

Fuente. Elaboración propia a partir del informe GEM España 2019-2020

Los datos aquí ofrecidos nos muestran que desde la perspectiva de género hay más empresas femeninas que masculinas jóvenes (65,9\% frente al 63,9\%) y maduras $(83,8 \%$ frente al $83,1 \%)$ sin innovación. Y son más las empresas masculinas que presentan alguna innovación $(25,8 \%$ en las iniciadas y $10,7 \%$ en las consolidadas) que las femeninas ( $19,1 \%$ en las iniciadas y $9,8 \%$ en las consolidadas). En ambos casos existe un mayor porcentaje de innovación en las empresas jóvenes; evidenciándose, además, que las nuevas empresas femeninas son completamente innovadoras un $5 \%$ más que las masculinas, aunque este porcentaje disminuye a más de la mitad en las empresas consolidadas (6,4\% femeninas y $6,2 \%$ masculinas). Carrillo et al. (2019) consideran que esta situación puede deberse a que actualmente las empresas que están surgiendo cuentan con equipos de trabajo multidisciplinares 
que poseen más sensibilización y formación en competencias creativas e innovadoras.

\section{El emprendimiento desde la tecnología}

Es importante analizar también el uso de las tecnologías en las empresas, lo que influye en la identificación de su grado de innovación. En este sentido, podemos diferenciar entre emprendimiento tecnológico (nuevos productos basados en el avance en la investigación), emprendimiento tecnológico digital (nuevos productos basados solo en tecnologías de la comunicación) y emprendimiento digital (nuevos productos y servicios basados en internet) (Tejeiro-Koller et al., 2021).

En la Tabla 4 se muestra el porcentaje del uso y grado de novedad de las tecnologías utilizadas por emprendedoras y emprendedores, tanto en fase inicial como en fase consolidada.

Tabla 4

Uso y grado de novedad de las tecnologías en las empresas españolas desde la perspectiva de género

\begin{tabular}{cllllllll}
\hline \multirow{2}{*}{$\begin{array}{c}\text { Tipo de } \\
\text { empresa }\end{array}$} & Género & \multicolumn{3}{c}{ Uso de las Tecnologías } & \multicolumn{3}{c}{$\begin{array}{c}\text { Grado de novedad de las } \\
\text { Tecnologías }\end{array}$} \\
\cline { 2 - 8 } & & Bajo & Medio & Alto & $\begin{array}{l}\text { Menos de } \\
1 \text { año }\end{array}$ & $1-5$ años & $\begin{array}{l}\text { Más de } \\
5 \text { años }\end{array}$ \\
\hline \multirow{2}{*}{ Iniciadas } & Mujer & $95 \%$ & $3,2 \%$ & $1,8 \%$ & $9,4 \%$ & $18,5 \%$ & $72,1 \%$ \\
\hline \multirow{2}{*}{ Consolidadas } & Hombre & $85,9 \%$ & $6 \%$ & $8 \%$ & $10,5 \%$ & $20,5 \%$ & $69 \%$ \\
\cline { 2 - 8 } & Mujer & $92 \%$ & $6,2 \%$ & $1,7 \%$ & $5,5 \%$ & $9,2 \%$ & $85,4 \%$ \\
\cline { 2 - 8 } & Hombre & $88,4 \%$ & $7,3 \%$ & $4,3 \%$ & $6,2 \%$ & $14,5 \%$ & $79,3 \%$ \\
\hline
\end{tabular}

Fuente. Elaboración propia a partir del informe GEM España 2019-2020

Los datos de la Tabla 4 nos indican que las empresas femeninas hacen un bajo uso de la tecnología (en las iniciadas un 95\% y en las consolidadas un $92 \%$ ) y muy pocas presentan una alta utilización de la misma (menos de un $2 \%$ en ambas tipologías). En las empresas masculinas, la situación se repite en el primero de los casos, aunque en menor porcentaje que en las empresas femeninas. Sin embargo, en cuanto a la alta utilización, las empresas masculinas sobresalen incluso más en las iniciadas (8\%) que en las consolidadas (4,3\%).

Es significativo resaltar, a este respecto, que a pesar de que las empresas masculinas usan más las tecnologías que las femeninas, son estas últimas las que poseen un mayor grado de novedad mantenido en el tiempo (más de 5 años). Esta realidad se evidencia tanto en el caso de las nuevas empresas (72,1\% frente al $69 \%)$, como en las ya consolidadas (85,4\% frente al $79,3 \%)$. Situación que difiere con relación a los primeros años, donde el grado de novedad es algo más destacado en las empresas masculinas jóvenes (10,5\% frente al 9,4\% - menos de 1 año - y 20,5\% frente al 18,5 - entre 1 y 5 años -) y en las empresas masculinas maduras (6,2\% frente al 5,5\% - menos de 1 año - y 14,5\% frente al 9,2\% - entre 1 y 5 años -). El significado 
de estos datos puede estar en que los hombres utilizan las tecnologías como un fin y las mujeres, sin embargo, como un medio para conseguir sus propósitos profesionales y personales (ONU Mujeres, 2021).

\section{El emprendimiento desde la internacionalización}

En último término vamos a hacer referencia a la internacionalización de las empresas españolas. Como se aprecia en la Tabla 5, existe poca internacionalización, tanto en las empresas jóvenes como en las maduras. No obstante, esta situación se evidencia ligeramente más en las empresas consolidadas (femeninas un $76,1 \%$ y masculinas un $72,6 \%$ ) que en las iniciadas (femeninas un $72,2 \%$ y masculinas un $66,8 \%)$.

Tabla 5

Grado de internacionalización en las empresas españolas desde la perspectiva de género

\begin{tabular}{clllll}
\hline \multirow{2}{*}{$\begin{array}{c}\text { Tipo de } \\
\text { empresa }\end{array}$} & \multirow{2}{*}{ Género } & \multicolumn{4}{c}{ Grado de internacionalización } \\
\cline { 2 - 6 } Iniciadas & Mujer & $1,9 \%$ & $5,9 \%$ & $20 \%$ & $72,2 \%$ \\
\cline { 2 - 6 } & Hombre & $3,8 \%$ & $6,9 \%$ & $22,6 \%$ & $66,8 \%$ \\
\hline \multirow{2}{*}{ Consolidadas } & Mujer & $1,2 \%$ & $2 \%$ & $20,7 \%$ & $76,1 \%$ \\
\cline { 2 - 6 } & Hombre & $1,4 \%$ & $5,3 \%$ & $20,7 \%$ & $72,6 \%$ \\
\hline
\end{tabular}

Fuente. Elaboración propia a partir del informe GEM España 2019-2020

Con relación a las empresas femeninas iniciadas, estas presentan un menor porcentaje en todos los grados de internacionalización frente a las masculinas $(27,8 \%$ y $33,2 \%$ respectivamente). Esto mismo sucede con las empresas consolidadas (23,9\% en las empresas de mujeres y $27,4 \%$ en las empresas de hombres).

Por otra parte, existen más empresas femeninas jóvenes que poseen el $75 \%$ de internacionalización $(1,9 \%)$ que maduras $(1,2 \%)$. Ocurre lo mismo con las que poseen entre el $25 \%$ y el $75 \%$ de internacionalización (jóvenes $5,9 \%$ y maduras $2 \%$ ). Sin embargo, existe una pequeña diferencia en aquellas empresas creadas por mujeres con menos del $25 \%$ de internacionalización, en las que destacan las consolidadas $(20,7 \%)$ frente a las iniciadas $(20 \%)$.

Podemos concluir, a este respecto, que el mayor porcentaje de empresas con grado de internacionalización, tanto masculinas como femeninas, se sitúa en menos de un $25 \%$. Esto nos hace pensar que el emprendimiento español busca su zona de confort en el país de origen donde se asumen menos riesgos y existen menos cotas de competitividad que en el entorno internacional, lo que sin embargo merma posibilidades de transferir su potencial a otros países (Ramos, 2017). 


\section{Conclusiones}

Desde el año 2000 hasta nuestros días, el emprendimiento en España ha tenido una evolución muy positiva gracias a las políticas sociales y educativas que lo han fomentado, posicionando a nuestro país entre los estados europeos que más oportunidades ofrecen para su desarrollo (Peña et al., 2020).

No obstante, si unimos la variable emprendimiento a la de género, existen ciertos factores que determinan el desarrollo del emprendimiento femenino y que influyen en su expansión. Nos referimos, por un lado, a los factores estructurales y sociales, los cuales indican que las mujeres se interesan más por el emprendimiento social, aquel al que tradicionalmente han estado abocadas y que les permite compaginar mejor su vida profesional y personal; requieren competencias tecnológicas y empresariales que deberían desarrollarse a través de la educación formal (primaria, secundaria y universitaria); y necesitan referencias concretas de emprendedoras de éxito que les sirvan de modelo y rompan el "techo de cristal" que les impide tener experiencias previas de liderazgo y gestión. Por otro lado, están los factores cognitivos-emocionales, los cuales nos hablan de mujeres competentes que no se perciben así, con miedo al fracaso, lo que debilita sus negocios y revierte en la creación de empresas más por necesidad que por oportunidad. Con motivaciones y aspiraciones encaminadas a "ganarse la vida", aunque poco a poco "busquen cubrir un hueco en el mercado", así como "crear riqueza y marcar diferencias", poniendo en marcha negocios más innovadores y startups. A estos factores se unen los que impulsan o bloquean el emprendimiento femenino, que son contextuales e incluyen el marco institucional del que deben provenir políticas activas de potenciación empresarial, el entorno internacional, nacional, regional y local, que debe promover espacios de encuentro y vías de apoyo (formación, financiación y recursos), y el entorno familiar, cuyo apoyo moral y desinteresado es fundamental para que una mujer se sienta acompañada en la aventura de emprender.

Estos factores son determinantes para las empresas españolas, tanto para las de reciente creación como para las ya consolidadas, como hemos podido comprobar al analizar los Informes GEM publicados hasta la fecha de entrega de este artículo, y actualmente aún más ante la situación derivada de la crisis del COVID19, la cual va a requerir una reactivación y transformación del emprendimiento, especialmente del femenino y con impacto social, a través de las siguientes iniciativas, punto de partida de futuras investigaciones (Fuentes y Neira, 2021):

- Diseño de programas de acompañamiento para emprendedoras.

- Orientación para percibir nuevas oportunidades y modelos de negocio.

- Promoción del modelo Entrepreneurial Universities, buscando nuevas sinergias entre el ecosistema emprendedor, el corporativo y el académico. 
- Presencia de los Objetivos de Desarrollo Sostenible en el nuevo escenario socioeconómico.

- Desarrollo de infraestructuras y formación tecnológica, potenciando la digitalización y la innovación.

- Trabajo en red de todos los agentes que intervienen en el ecosistema emprendedor.

\section{Referencias}

Aido-Almagro, B., Diánez-González, J.P., Camelo-Ordaz, C. y Ruiz-Navarro, J. (2016). Identificación del emprendimiento de alto potencial. Un análisis sobre el reconocimiento de oportunidad en diferentes economías. Enfoques, 399, 7584.

Aguilar, A.Y., Vargas, A., Hernández L.G y Lugo M. Á. Identificación de antecedentes familiares de Emprendimiento y su incidencia en la decisión de Emprender. ANFEI Digital, 12, 1-11.

Álvarez, M.J., Rebollo, M.A. y Rodríguez, M.R. (2013). Factores facilitadores y bloqueadores del emprendimiento femenino: orientar para el emprendimiento desde una perspectiva de género. Investigación e innovación educativa. Recuperado de: https://core.ac.uk/download/pdf/157763941.pdf

Álvarez, C., Noguera, M. y Urbano, D. (2012). Condicionantes del entorno y emprendimiento femenino. Un estudio cuantitativo en España. Universidad de Medellín. Recuperado de: https://repository.udem.edu.co/handle/11407/3329

Arcos, P.G. (2020). Aplicación y beneficios del plan de negocios para la creación y consolidación de microempresas [Tesis de pregrado, Universidad Técnica del Norte]. Recuperado de: http://repositorio.utn.edu.ec/handle/123456789/10741

Arenius, P. y Minniti, M. (2005). Perceptual variables and nascent entrepreneurship. Small Business Economic, 24, 223-247. http://dx.doi.org/10.1007/s11187-0051984-x.

Barney, J. (1991). Firm Resources and Sustained Competitive Advantage. Journal of Management, 17(1), 99-120.

Bisquerra, R. (Coord.). (2009). Metodología de investigación educativa. La Muralla.

Brüderl, J., Preisendörfer, P. y Ziegler, R. (1992) Survival Chances of Newly Founded Business Organizations. American Sociological Review, 2(57), 227-242. https://doi.org/10.2307/2096207

Brush, C.G. (1992) Research on women business owners: past trends, a new perspective and future directions. Entrepreneurship Theory and practice, 4(16), 5-30. https://doi.org/10.1177/104225879201600401

Carrillo, A., Caballero, F.C. y Roque, R.V. (2019). Formación en creatividad e innovación: estudiando las percepciones y prácticas de los jóvenes emprendedores locales. Revista Iberoamericana de Contaduría, Economía y Administración: RICEA, 8(15), 1-28. 
De la O Cordero, D. y Monge-González, R. (2019). Factores que influyen en el desempeño de las PYME: una perspectiva cualitativa de los empresarios costarricenses. Revista Academia y Negocios. R.A.N., 4(2), 95-106.

DeTienne, D. y Chandler, G. (2007) The Role of Gender in Opportunity Identification. Entrepreneurship Theory and Practice, 31, 365-386.

https://doi.org/10.1111/j.1540-6520.2007.00178.x.

Díaz, M.C. y Carter, S. (2009). Resource mobilization through business owner's networks: is gender an issue? International Journal of Gender and Entrepreneurship, 1(3), 226-252.

https://doi.org/10.1108/17566260910990919

Elizundia, $M^{a}$.E. (2015). Desempeño de nuevos negocios: perspectiva de género. Contaduría y Administración, 60(2), 468-485. https://doi.org/10.1016/S01861042(15)30010-3

Fuentes Ma‥M. y Neira, I. (Dirs.).(2021). Situación del emprendimiento en España ante la crisis del COVID-19 Análisis y recomendaciones. Observatorio del Emprendimiento de España.

Figueroa, C., De La Fuente, C. y Segovia, M. (2011). La contribución de una SGR al emprendimiento de mujeres: el caso de Avalmadrid. BURJC Digital. Universidad Rey Juan Carlos.

Galindo, M.A. y Méndez, M.T. (2011). La actividad emprendedora y competitividad: Factores que inciden sobre los emprendedores. Papeles de Europa, 22, 6175. http://dx.doi.org/10.5209/rev PADE.2011.v22.3

García-Villalobos, J.C., Villaseca-Morales, D. y González-Pérez, S. (2019). Emprendimiento femenino y financiación social: un estudio comparado. REVESCO. Revista De Estudios Cooperativos, 132, 97-121. http://dx.doi.org/10.5209/REVE.65974

Gordon, I. y Jack, S. (2010) HEl engagement with SME: developing social capital. International Journal of Entrepreneurial Behavior \& Research, 6(16), 517- 539. https://doi.org/10.1108/13552551011082489

Heilbrunn, S. (2004) Impact of Gender on Difficulties Faced by Entrepreneurs. The International Journal of Entrepreneurship and Innovation, 5(3),159-165. https://doi.org/10.5367/0000000041513420

Hoang, H. y Antoncic, B. (2003) Network-based research in entrepreneurship: A critical review. Journal of Business Venturing, 2(18), 165-187.

https://doi.org/10.1016/S0883-9026(02)00081-2

Macías, D., Santacruz-Valencia, L.P. y Gómez, J. (2021). La importancia de enseñar secuenciación en edades tempranas: una puerta al desarrollo de competencias STEM. IE Comunicaciones: Revista Iberoamericana de Informática Educativa, 33, 31-42.

Maldonado, B.R., Lara, G.J. y Maya, A.M. (2016). La Mujer como motor del desarrollo local: Una experiencia puntual. Revista San Gregorio, 1(2), 91-107. http://dx.doi.org/10.36097/rsan.v0i0.93 
Montero-González, B. y Camacho-Ballesta, J.A. (2018). Caracterización del emprendimiento femenino en España: Una visión de conjunto. REVESCO. Revista De Estudios Cooperativos,129, 39-65.

http://dx.doi.org/10.5209/REVE.61936

OCDE. (2013). Cerrando las brechas de género: es hora de actuar. CIEDESS.

ONU Mujeres (2021) Innovación y tecnología. https://www.unwomen.org/es

Peña, I., Guerrero, M., González-Pernía, J.L. y Montero, J. (2020). Global Entrepreneurship Monitor. Informe GEM España 2019-2020. Universidad de Cantabria, Asociación RED GEM España y CISE.

Peña, I., Guerrero, M., González-Pernía, J.L. y Montero, J. (2019). Global Entrepreneurship Monitor. Informe GEM España 2018-2019. Universidad de Cantabria, Asociación RED GEM España y CISE.

Peña, I., Guerrero, M., González-Pernía, J.L. y Montero, J. (2018). Global Entrepreneurship Monitor. Informe GEM España 2017-2018. Universidad de Cantabria, Asociación RED GEM España y CISE.

Ramos, E.B. (2017). La internacionalización de las empresas españolas por medio de alianzas estratégicas: factores de éxito vinculados al comportamiento de los aliados. (Trabajo Fin de Grado Inédito). Universidad de Sevilla.

Santander-Astorga, P., Fernández-Robin, C. y Yáñez-Martínez, D. (2016). Motivaciones y condicionantes contextuales en el emprendimiento liderado por mujeres chilenas. Revista de Ciencias Sociales (Ve), XXII(2), 63-77.

South Summit. (2019). Mapa del emprendimiento. Spain Startup South Summit y IE University.

Tejeiro-Koller, M., Molina-López, M.Ma y García-Villalobos, J.C. (2021). Emprendimiento digital femenino para el desarrollo social y económico: características y barreras en España. REVESCO. Revista De Estudios Cooperativos, 138, 1-13. https://dx.doi.org/10.5209/REVE.75561

Terán, E.F. y Guerrero, A.M. (2019). ¿Emprendimiento por oportunidad o por necesidad? Estudio comparativo entre países. Mikarimin. Revista Científica Multidisciplinaria, 5(2), 77-86.

Themudo, N.S. (2009). Gender and the Nonprofit sector. Nonprofit and Voluntary Sector Quarterly, 4(38), 663-683. https://doi.org/10.1177/0899764009333957

Vélez-Romero, X. y Ortiz-Restrepo, S. (2016). Emprendimiento e innovación: una aproximación teórica. Dominio de las Ciencias, 2(4), 346-369

Verheul, I., Thurik, R., Hessels, J. y Zwan P. (2016). Factors influencing the entrepreneurial engagement of opportunity and necessity entrepreneurs. Eurasian Business Review, 6(3), 273-295. https://doi.org/10.1007/s40821-016$\underline{0065-1}$

Zhao, H., Seibert, S.E. y Hills, G.E. (2005) The Mediating Role of Self-Efficacy in the Development of Entrepreneurial Intentions. The Journal of applied psychology, 90(6), 1265-1272. https://doi.org/10.1037/0021-9010.90.6.1265. 
